\title{
Factors related to outcome of bloodstream infections due to Candida parapsilosis complex
}

\author{
Francesco Barchiesi ${ }^{1^{*}}$ (D), Elena Orsetti ${ }^{1}$, Patrizia Osimani ${ }^{3}$, Carlo Catassi ${ }^{2}$, Fabio Santelli ${ }^{4}$ and Esther Manso ${ }^{5}$
}

\begin{abstract}
Background: Although Candida albicans is the most common cause of fungal blood stream infections (BSIs), infections due to Candida species other than C. albicans are rising. Candida parapsilosis complex has emerged as an important fungal pathogen and became one of the main causes of fungemia in specific geographical areas. We analyzed the factors related to outcome of candidemia due to C. parapsilosis in a single tertiary referral hospital over a five-year period.
\end{abstract}

Methods: A retrospective observational study of all cases of candidemia was carried out at a 980-bedded University Hospital in Italy. Data regarding demographic characteristics and clinical risk factors were collected from the patient's medical records. Antifungal susceptibility testing was performed and MIC results were interpreted according to CLSI species-specific clinical breakpoints.

Results: Of 270 patients diagnosed with Candida BSIs during the study period, 63 (23\%) were infected with isolates of $C$. parapsilosis complex which represented the second most frequently isolated yeast after C. albicans. The overall incidence rate was 0.4 episodes/1000 hospital admissions. All the strains were in vitro susceptible to all antifungal agents. The overall crude mortality at 30 days was $27 \%(17 / 63)$, which was significantly lower than that reported for C. albicans BSIs (42\% [61/146], $p=0.042$ ). Being hospitalized in ICU resulted independently associated with a significant higher risk of mortality (HR 4.625 [C195\% 1.015-21.080], $p=0.048$ ). Conversely, early CVC removal was confirmed to be significantly associated with a lower risk of mortality (HR 0.299 [Cl95\% 0.102-0.874], $p=0.027$ ). Finally, the type of primary antifungal therapy did not influence the outcome of infection.

Conclusions: Candidemia due to C. parapsilosis complex, the second most commonly causative agent of yeast BSIs in our center, is characterized by a non-negligible mortality at 30 days. An early CVC removal is associated with a significant reduced mortality.

Keywords: Candida paraspilosis complex, Candidemia, Risk factors, Mortality, Antifungal agents

\section{Background}

Candida is an important cause of bloodstream infections (BSIs) and it is the main agent of invasive fungal infection in hospitalized patients [1, 2]. Although Candida albicans is the most common cause of invasive candidiasis, infections due to Candida species other than C. albicans are rising [3, 4]. In particular, Candida parapsilosis complex, which includes C. parapsilosis

\footnotetext{
* Correspondence: f.barchiesi@univpm.it

${ }^{1}$ Clinica Malattie Infettive; Università Politecnica delle Marche, Azienda Ospedaliero-Universitaria, Ospedali Riuniti Umberto I- G.M. Lancisi - G. Salesi, Ancona, Italy

Full list of author information is available at the end of the article
}

sensu strictu (the most frequent), Candida orthopsilosis and Candida metapsilosis, has emerged as an important fungal pathogen and became one of the main causes of fungemia in tertiary-care hospitals [5-9]. Although the mortality rate due to this species is generally lower than that reported for other Candida species, C. parapsilosis complex possesses several distinct features, such as its ability to develop biofilms on intravascular devices, high affinity for parenteral nutrition, and an intrinsic low susceptibility to echinocandins [10-12]. 
Table 1 Comparison of epidemiological, clinical characteristics and outcome of patients with BSIs due to Candida parapsilosis and Candida albicans

\begin{tabular}{|c|c|c|c|}
\hline \multirow[t]{2}{*}{ Variables } & \multicolumn{3}{|c|}{ Patients with BSIs due to: } \\
\hline & C. parapsilosis $(n=63)$ & C. albicans $(n=146)$ & $P$ value $^{a}$ \\
\hline$\overline{\text { Age (years), median (IQR) }}{ }^{b}$ & $67(43-76)$ & $51(66-77)$ & 0.133 \\
\hline Neonates (<3 months) & $1(2)$ & $5(3)$ & 0.465 \\
\hline Elderly (>65 years) & $32(51)$ & $72(49)$ & 0.844 \\
\hline Male sex, $n(\%)$ & $35(56)$ & $91(63)$ & 0.358 \\
\hline \multicolumn{4}{|l|}{ Ward } \\
\hline Internal Medicine, $n$ (\%) & $25(40)$ & $52(36)$ & \multirow[t]{3}{*}{0.448} \\
\hline Surgery, $n(\%)$ & $17(27)$ & $32(22)$ & \\
\hline Intensive Care Unit, $n$ (\%) & $21(33)$ & $62(43)$ & \\
\hline Comorbidities, $n$ (\%) & $57(90)$ & $137(94)$ & 0.387 \\
\hline Chronic pulmonary diseases, $n(\%)^{c}$ & $9(14)$ & $11(8)$ & 0.127 \\
\hline Haematological malignancy, n (\%) & $1(2)$ & $8(5)$ & 0.203 \\
\hline Cardiovascular diseases, $n(\%)^{d}$ & $27(43)$ & $67(46)$ & 0.685 \\
\hline Neurological diseases, $n(\%)^{e}$ & $10(16)$ & $24(16)$ & 0.919 \\
\hline Gastrointestinal diseases, $n(\%)^{f}$ & $21(33)$ & $43(29)$ & 0.576 \\
\hline Diabetes mellitus, $n$ (\%) & $8(13)$ & $28(19)$ & 0.254 \\
\hline Solid tumors, $n(\%)$ & $15(24)$ & $43(30)$ & 0.403 \\
\hline Chronic renal failure, n (\%) & $7(11)$ & $12(8)$ & 0.504 \\
\hline Previous surgery (<30 days), $n$ (\%) & $32(51)$ & $67(46)$ & 0.514 \\
\hline Gastrointestinal surgery, $n$ (\%) & $8(13)$ & $19(13)$ & 0.950 \\
\hline Cardiovascular surgery, n (\%) & $13(21)$ & $28(19)$ & 0.807 \\
\hline Other surgery, $n(\%)^{g}$ & $12(19)$ & $24(16)$ & 0.646 \\
\hline Central venous catheter, $n$ (\%) & $56(89)$ & $127(87)$ & 0.702 \\
\hline BSI CVC-related, $n$ (\%) & $36(64)$ & $57(45)$ & 0.015 \\
\hline Other devices, $n(\%)^{h}$ & $57(90)$ & $129(88)$ & 0.653 \\
\hline Previous invasive procedures $(<72 \mathrm{~h}), n(\%)^{i}$ & $16(25)$ & $44(30)$ & 0.486 \\
\hline Parenteral nutrition, $n$ (\%) & $43(68)$ & $105(72)$ & 0.592 \\
\hline Immunosuppressive therapy, $n(\%)^{j}$ & $18(29)$ & $56(34)$ & 0.174 \\
\hline Neutropenia, $n$ (\%) & $4(6)$ & $7(5)$ & 0.644 \\
\hline Septic shock, n (\%) & $2(3)$ & $12(8)$ & 0.180 \\
\hline Prior antibiotic therapy, $n$ (\%) & $58(92)$ & $139(95)$ & 0.370 \\
\hline Previous antifungal therapy (<30 days), $n$ (\%) & $5(8)$ & $21(14)$ & 0.195 \\
\hline Concomitant bacteriemia, $n$ (\%) & $15(24)$ & $40(27)$ & 0.588 \\
\hline Other coinfections, $n(\%)^{k}$ & $39(62)$ & $89(61)$ & 0.897 \\
\hline Appropriate antifungal therapy, $n(\%)^{\prime}$ & $38(60)$ & $92(63)$ & 0.712 \\
\hline Primary azole therapy & $41(65)$ & $77(53)$ & 0.098 \\
\hline Primary echinocandin therapy & $7(11)$ & $34(23)$ & 0.041 \\
\hline Primary polyene therapy & $1(2)$ & $3(2)$ & 0.820 \\
\hline None & $14(22)$ & $32(22)$ & 0.961 \\
\hline
\end{tabular}


Table 1 Comparison of epidemiological, clinical characteristics and outcome of patients with BSIs due to Candida parapsilosis and Candida albicans (Continued)

\begin{tabular}{llll}
\hline Overall mortality, $n(\%)$ & $17(27)$ & $61(42)$ & 0.042 \\
Early mortality (days 1-7), $n(\%)$ & $4(6)$ & $24(17)$ & 0.049 \\
Late mortality (days 8-30), $n(\%)$ & $13(21)$ & $37(25)$ & 0.464 \\
\hline
\end{tabular}

${ }^{a}$ Comparisons between groups were performed using Wilcoxon rank sum test for quantitative variables and Chi-Square test (or Fisher Exact Test when expected frequencies were less than five) for qualitative variables

${ }^{\mathrm{b}} \mathrm{IQR}$, Interquartile range

${ }^{c}$ Chronic pulmonary diseases include asthma, chronic bronchitis, emphysema and lung fibrosis

${ }^{\mathrm{d} C a r d i o v a s c u l a r}$ diseases include heart failure, ischemic heart disease, endocarditis and arrhythmia

eNeurological diseases include Parkinson's disease, Alzheimer's disease and paralysis

fGastrointestinal diseases include Crohn's disease, ulcerative colitis, chronic pancreatitis and gallbladder stones

${ }^{g}$ Other surgery includes plastic surgery, thoracic surgery, orthopaedic surgery, urological surgery and neurosurgery

h Other devices include urinary catheter, surgical drainage, cutaneous gastrostomy and tracheostomy tube

'Previous invasive procedures include endoscopy and positioning of any device

${ }^{j}$ Immunosuppressive therapy include corticosteroids, calcineurin inhibitors and monoclonal antibodies

${ }^{k}$ Other coinfections include bacterial and/or fungal infections in sites other than blood

'Appropriate antifungal therapy was considered when the appropriate drug with adequate dosage was started $\leq 72 \mathrm{~h}$ from the first blood culture performed

The aim of this study was to analyze the factors related to outcome of candidemia due to C. parapsilsosis complex in a single institution over a five-year period.

\section{Methods}

\section{Study population and data collection}

A retrospective observational study of all cases of candidemia was carried out from January 1, 2010 to December 31, 2014 (5-year period) in a single 980-bedded referral University Hospital in Ancona, Italy. A case of Candida BSI was defined as a peripheral isolation of Candida species from blood culture in a patient with temporally related clinical signs and symptoms of infection. All Candida BSIs were identified through the microbiological laboratory database. Data regarding demographic characteristics and clinical risk factors were collected from the patient's medical records. Appropriate antifungal therapy was considered when an appropriate drug (based on subsequent in vitro susceptibility testing results) with adequate dosage was started within $72 \mathrm{~h}$ from the first blood culture performed. Adequate dosage of an antifungal agent was defined according to IDSA 2009 guidelines [13]. Early central venous catheter $(\mathrm{CVC})$ removal was defined a removal of the line within $48 \mathrm{~h}$ from drawing blood culture. Mortality was calculated after 7 and 30 days from the occurrence of the episode of Candida BSI.

Candida species were isolated from blood samples using BacT/ALERT (bioMérieux) and identified with standard techniques. Each of the three species of $C$. parapsilosis sensu lato was identified using the MALDITOF Biotyper ${ }^{\mathrm{Tw}}$ [14]. Antifungal susceptibility testing was performed using the SensitreYeastOne colorimetric plate (SYO) (Trek Diagnostic System) and MIC results were interpreted according to latest species-specific CBPs as established by the CLSI [15].

The present research has been performed in accordance with the ethical standards of the 1964 Declaration of Helsinki and its later amendements. The Institutional
Review Board of the Azienda Ospedaliero-Universitaria Ospeadali Riuniti Umberto $I^{\circ}$-Lancisi-Salesi granted retrospective access to the data without need for individual informed consent.

\section{Statistical analysis}

Quantitative data were shown as the median with interquartile ranges (Q1-Q3). Qualitative variables were expressed as absolute and relative frequencies. Categorical variables were compared using the $\chi^{2}$ test, whereas Mann-Whitney $U$ test or Fisher exact test were applied for continuous variables. Variables with a $p \leq 0.05$ at the descriptive analysis were analyzed by Cox regression. All statistical analyses were performed using the statistical package SPSS for Windows v. 20 (SPSS Inc., Chicago, IL, USA). A $P$ value of $\leq 0.05$ was considered to represent statistical significance and all statistical tests were two-tailed.

\section{Results}

Of 270 patients diagnosed with Candida BSIs during the study period, 63 (23\%) were infected with isolates of $C$. parapsilosis complex (95 C. parapsilosis sensu lato, 3 C. orthopsilsosis and $2 \%$ C. metapsilosis). This species represented the second most frequently isolated yeast after C. albicans (146 [54\%]). The remaining infections were due to C. tropicalis (23 [9]), C. glabrata (10 [4]) and other Candida species (10 [4 \%]). Although C. albicans represented the most commonly isolated species, its percentage significantly decreased from $68 \%$ to $48 \%$ in the time period considered $(p=0.040)$. On the opposite, there was a significant increase of the percentage of $C$. parapsilosis isolation from $8 \%$ (2010) to $30 \%$ (2014) $(p=0.036)$. The overall incidence rate of BSIs due to C. parapsilosis was 0.4 episodes $/ 1000$ hospital admissions.

Demographics and clinical characteristics of 63 patients infected with isolates of C. parapsilosis complex were compared with those of 146 patients infected with 
Table 2 Outcome of 63 patients with BSIs due to Candida parapsilosis complex considered in this study

\begin{tabular}{|c|c|c|c|}
\hline \multirow[t]{2}{*}{ Characteristics } & \multicolumn{3}{|l|}{ 30-day outcome } \\
\hline & Survival $(n=46)$ & Death $(n=17)$ & $P$ value $^{a}$ \\
\hline Age (years), median (IQR) ${ }^{b}$ & $62(31-75)$ & $76(63-78)$ & 0.046 \\
\hline Male sex, $n(\%)$ & $24(52)$ & $11(65)$ & 0.374 \\
\hline \multicolumn{4}{|l|}{ Ward } \\
\hline Internal Medicine, n (\%) & $21(46)$ & $4(24)$ & \multirow[t]{3}{*}{0.033} \\
\hline Surgery, $n(\%)$ & $14(30)$ & $3(18)$ & \\
\hline Intensive Care Unit, $n$ (\%) & $11(24)$ & $10(59)$ & \\
\hline Comorbidities, $n$ (\%) & $40(87)$ & $17(100)$ & 0.117 \\
\hline Chronic pulmonary diseases, $n(\%)^{c}$ & $5(11)$ & $4(24)$ & 0.235 \\
\hline Haematological malignancy, n (\%) & $1(2)$ & $0(0)$ & 0.540 \\
\hline Cardiovascular diseases, $n(\%)^{d}$ & $15(33)$ & $12(71)$ & 0.006 \\
\hline Neurological diseases, $n(\%)^{e}$ & $9(20)$ & $1(6)$ & 0.263 \\
\hline Gastrointestinal diseases, $n(\%)^{f}$ & $16(35)$ & $5(29)$ & 0.688 \\
\hline Diabetes mellitus, $n$ (\%) & $4(9)$ & $4(24)$ & 0.195 \\
\hline Solid tumors, $n(\%)$ & $9(20)$ & $6(35)$ & 0.193 \\
\hline Chronic renal failure, $n$ (\%) & $5(11)$ & $2(12)$ & 1.000 \\
\hline Previous surgery (<30 days), $n$ (\%) & $23(50)$ & $9(53)$ & 0.835 \\
\hline Gastrointestinal surgery, $n$ (\%) & $5(11)$ & $3(18)$ & 0.671 \\
\hline Cardiovascular surgery, n (\%) & $7(15)$ & $6(35)$ & 0.080 \\
\hline Other surgery, $n(\%)^{9}$ & $11(24)$ & $1(6)$ & 0.154 \\
\hline Central venous catheter, $n$ (\%) & $41(89)$ & $15(88)$ & 0.920 \\
\hline BSI CVC-related, $n$ (\%) & $25(61)$ & $11(73)$ & 0.460 \\
\hline Early central venous catheter removal, $n(\%)^{h}$ & $41(100)$ & $11(73)$ & $<0.0001$ \\
\hline Other devices, $n(\%)^{i}$ & $40(87)$ & $17(100)$ & 0.178 \\
\hline Previous invasive procedures $(<72 \mathrm{~h}), n(\%)^{j}$ & $9(20)$ & $7(41)$ & 0.080 \\
\hline Parenteral nutrition, $n$ (\%) & $29(63)$ & $14(82)$ & 0.143 \\
\hline Immunosuppressive therapy, $n(\%)^{k}$ & $13(28)$ & $5(29)$ & 0.920 \\
\hline Neutropenia, $n$ (\%) & $3(7)$ & $1(6)$ & 1.000 \\
\hline Septic shock, n (\%) & $1(2)$ & $1(6)$ & 0.470 \\
\hline Prior antibiotic therapy, $n$ (\%) & $42(91)$ & $16(94)$ & 0.713 \\
\hline Previous antifungal therapy, $n$ (\%) & $4(9)$ & $1(6)$ & 0.713 \\
\hline Concomitant bacteriemia, $n$ (\%) & $12(26)$ & $3(18)$ & 0.487 \\
\hline Other coinfections, $n(\%)^{\prime}$ & $28(61)$ & $11(65)$ & 0.780 \\
\hline
\end{tabular}


Table 2 Outcome of 63 patients with BSIs due to Candida parapsilosis complex considered in this study (Continued)

\begin{tabular}{llll}
\hline Appropriate antifungal therapy $^{m}$ & $30(65)$ & $8(47)$ & 0.190 \\
Primary azole therapy & $32(70)$ & $5(53)$ & 0.219 \\
Primary echinocandin therapy & $5(11)$ & $2(12)$ & 1.000 \\
Primary polyene therapy & $1(2)$ & $6(0)$ & 1.000 \\
None & $8(17)$ & $6(35)$ & 0.129
\end{tabular}

${ }^{a}$ Comparisons between groups were performed using Wilcoxon rank sum test for quantitative variables and Chi-Square test (or Fisher Exact Test when expected frequencies were less than five) for qualitative variables

${ }^{\mathrm{b}} \mathrm{IQR}$, Interquartile range

${ }^{c}$ Chronic pulmonary diseases include asthma, chronic bronchitis, emphysema and lung fibrosis

${ }^{\mathrm{d} C a r d i o v a s c u l a r}$ diseases include heart failure, ischemic heart disease, endocarditis and arrhythmia

${ }^{\mathrm{e}}$ Neurological diseases include Parkinson's disease, Alzheimer's disease and paralysis

fGastrointestinal diseases include Crohn's disease, ulcerative colitis, chronic pancreatitis and gallbladder stones

${ }^{g}$ Other surgery includes plastic surgery, thoracic surgery, orthopaedic surgery, urological surgery and neurosurgery

${ }^{\mathrm{h}}$ Early CVC removal was considered occurring within $48 \mathrm{~h}$ from blood cultures drawing

i Other devices include urinary catheter, surgical drainage, cutaneous gastrostomy and tracheostomy tube

${ }^{j}$ Previous invasive procedures include endoscopy and positioning of any device

${ }^{\mathrm{k}}$ Immunosuppressive therapy include corticosteroids, calcineurin inhibitors and monoclonal antibodies

' Other coinfections include bacterial and/or fungal infections in sites other than blood

${ }^{\mathrm{m}}$ Appropriate antifungal therapy was considered when the appropriate drug with adequate dosage was started within $72 \mathrm{~h}$ the first blood culture performed

isolates of $C$. albicans and the results are shown in Table 1. Median patients age was 67 years. Male accounted for $56 \%$ of the population. Twenty-five isolates were recovered from patients hospitalized in medical wards, 21 from patients hospitalized in ICUs and 17 from patients hospitalized in surgical wards. The majority of patients $(90 \%)$ suffered from multiple comorbidities (range 2 to 4 ) being the most common cardiovascular (43) and gastrointestinal (33\%) diseases. A total of $51 \%$ of patients underwent a surgical intervention within 30 days from the onset of candidemia. In comparison with $C$. albicans, BSIs due to $C$. parapsilosis were significantly more often associated with the use of CVC $(p=0.015)$. The overall crude mortality at 30 days was higher in patients infected with $C$. albicans than $C$. parapsilosis (42\% vs $27 \%$, respectively, $p=0.042$ ).

Factors related to outcome of 63 patients with BSIs due to $C$. parapsilosis are reported in Table 2 . Older age $(p=0.046)$, being hospitalized in ICU or in medical wards rather than in surgical wards $(p=0.033)$, being recently diagnosed with a cardiovascular disease $(p=$ 0.006), and the lack of an early CVC removal $(p=0.003)$ were all factors associated with a significant higher probability of death at the descriptive analysis. On multivariate analysis, being hospitalized in ICU resulted independently associated with a significant higher risk of mortality (HR 4.625 [CI95\% 1.015-21.080], $p=0.048$ ). Conversely, early CVC removal was confirmed to be significantly associated with a lower risk of mortality (HR 0.299 [CI95\% 0.102-0.874], $p=0.027$ ).

Table 3 shows the results of antifungal susceptibility testing to nine antifungal agents as routinely performed by the SYO method which was developed to provide an easy alternative to the CLSI procedure. With the exception of one isolate that showed to be susceptible dose dependent to fluconazole (MIC $4 \mu \mathrm{g} / \mathrm{ml}$ ) and two isolates which were found to have non-wild type phenotype for flucytosine (MIC $1 \mu \mathrm{g} / \mathrm{ml}$ ), all isolates showed to be fully susceptible to all antifungals according to the CLSI interpretation [15].

Table 3 In vitro susceptibilities of Candida parapsilosis complex isolates considered in this study

\begin{tabular}{|c|c|c|c|c|c|c|c|c|c|}
\hline \multirow[t]{2}{*}{ Antifungal agents } & \multirow[t]{2}{*}{$\mathrm{MIC}$ range $(\mu \mathrm{g} / \mathrm{ml})$} & \multirow[t]{2}{*}{$\mathrm{MIC}_{50}(\mu \mathrm{g} / \mathrm{ml})$} & \multirow[t]{2}{*}{$\mathrm{MIC}_{90}(\mu \mathrm{g} / \mathrm{ml})$} & \multicolumn{6}{|c|}{$\%$ of isolates in the indicated category according to $\mathrm{CLSI}^{a}$} \\
\hline & & & & $\mathrm{S}$ & SDD & I & $\mathrm{R}$ & WT & Non-WT \\
\hline Amphotericin B & $\leq 0.12-1$ & 0.5 & 0.5 & - & - & - & - & 100 & 0 \\
\hline Flucytosine & $\leq 0.06-1$ & $\leq 0.06$ & 0.25 & - & - & - & - & 97 & 3 \\
\hline Fluconazole & $\leq 0.12-4$ & 0.25 & 1 & 98.4 & 1.6 & - & 0 & - & - \\
\hline Itraconazole & $\leq 0.015-0.25$ & 0.06 & 0.12 & - & - & - & - & 100 & 0 \\
\hline Voriconazole & $\leq 0.008-0.06$ & $\leq 0.008$ & 0.015 & 100 & 0 & - & 0 & - & - \\
\hline Posaconazole & $\leq 0.008-0.12$ & 0.03 & 0.06 & - & - & - & - & 100 & - \\
\hline Caspofungin & $0.06-1$ & 0.25 & 0.5 & 100 & - & 0 & 0 & - & - \\
\hline Anidulafungin & $0.12-2$ & 0.5 & 2 & 100 & - & 0 & 0 & - & - \\
\hline Micafungin & $0.12-2$ & 1 & 2 & 100 & - & 0 & 0 & - & - \\
\hline
\end{tabular}

${ }^{a}$ Category was interpreted according to CLSI breakpoints as reported in ref. 15 . S, susceptible; SDD susceptible dose dependent, $I$ intermediate, $R$ resistant, $W T$ wild type 


\section{Discussion and conclusions}

Although $C$. albicans remains the most common fungal isolate from blood, longitudinal studies showed a trend toward an increased prevalence of other Candida spp. with a larger proportion of C. glabrata in the United States and C. parapsilosis in some European (i.e.: Italy or Spain) and Latin American countries [3-9]. As far, few studies focused on the specific predictors influencing the outcome of BSIs due to C. parapsilosis [16-18].

First, we confirmed that the mortality due to this species is somewhat lower than that reported for C. albicans, having found a rate of $27 \%$ at 30 days. This figure is consistent with literature data in which the 30-day mortality due to this species ranges from $23 \%$ to $30 \%$ [14, 19-21].

Second, hospitalization in ICU showed to be independently associated with higher risk of mortality. Our data are in agreement with a large study showing a mortality rate increasing from $29 \%$ to $47 \%$ in patients with BSIs due to Candida spp. hospitalized, respectively, in non-ICU and ICU wards [22]. ICU stay would represent a surrogate marker of illness severity thereby facilitating the poor outcome of this population group.

Third, we showed that early CVC removal is protective in BSIs due to C. parapsilosis. Although the impact of CVC management has been extensively investigated on the outcome of patients with candidemia, few studies have examined this issue across individual Candida species. Two recent studies showed that CVC removal exerted a protective effect on the outcome of candidemia due to $C$. parapsilosis $[14,20]$. Since this species is characterized by a high propensity to develop biofilms on intravascular devices, their early removal play a fundamental role in determining the outcome of infection.

Fourth, we found that the outcome was not influenced by an appropriate antifungal therapy. Despite this finding is somewhat divergent from that reported by the current literature [23-25], there is a paucity of data considering the infection outcome of individual Candida species. In this regard, there are two important factors to consider. First, being $C$. parapsilosis less pathogenic than other Candida species (i.e.: C. albicans and C. tropicalis) [17], BSIs due to this entity could be more deeply affected by a correct general management (i.e.: prompt removal of any central line) rather than an early therapeutic intervention. Second, we defined appropriate antifungal therapy as the appropriate drug with adequate dosage started within $72 \mathrm{~h}$ from the first blood culture performed. Since C. parapsilosis has a time to positivity of blood cultures longer than those reported for C. albicans and C. tropicalis [26], preliminary blood culture showing the growth of yeast-like fungal pathogen could be delayed thereby determining an initial, although adequate, antifungal treatment after this time interval.
Interestingly, we found that primary antifungal treatment (i.e.: triazoles or echinocandins) did not influence the outcome of C. parapsilosis BSIs. Although, this Candida species possesses a natural low susceptibility profile to echinocandins [12] and there is still a debate on the use of these molecules in infections caused by this species, either randomized or not-randomized clinical trials have shown not significant differences in the success rates between arms [27-30]. Our data, although with a limited number of patients, corroborated these findings.

The present study have some limitations. First, being a single-center study, the number of patients considered is low. This feature has certainly weakened the statistical power of the study. Nevertheless, we have made all attempts to collect and analyze as many as clinical data as possible to reveal useful information for the management of patients infected with this Candida species. Second, being a retrospective study encompassing several departments and medical disciplines over a five years period, there was not a univocal management of each individual case. In this respect, serial follow-up blood cultures were not systematically performed and we were unable to include important parameters, other then 30day mortality, such as the persistence of positive blood cultures after the initiation of antifungal therapy.

In conclusion, our study shows that candidemia due to C. parapsilosis complex, the second most commonly causative agent of yeast BSIs in our center, is characterized by a non-negligible mortality at 30 days. While an early CVC removal is associated with significant reduced mortality, the type of primary antifungal therapy does not influence the outcome. Further prospective studies including higher number of patients are needed to corroborate these findings.

\section{Abbreviations}

BSIs, bloodstream infections; CBP, clinical breakpoints; CLSI, Clinical and Laboratory Standards Institute; CVC, central venous catheter; HR, hazard ratio; ICUs, Intensive Care Units; IDSA, Infectious Disease Society of America; MALDI-TOF, Matrix Assisted Laser Desorption/lonization Time of Flight Mass Spectrometry; MICs, minimum inhibitory concentrations; Q1-Q3, interquartile range; spp, species; h, hours.; SPSS, Statistical Package for the Social Sciences; SYO, SensitreYeastOne colorimetric plate

\section{Availability of data and materials}

The data cannot be shared as local Institutional Review Board has no policy to share the data without prior permission.

\footnotetext{
Authors' contributions

FB designed the study, analyzed data and wrote the article. EO, performed the acquisition of data, analyzed data and wrote the article. PO, CC, and FS, provided the clinical data. EM, provided the laboratory data. All authors drafted the article, revised it critically for important intellectual content, and approved the final article.
}

\section{Competing of interests}

The authors declare that they have no competing interests. The authors alone are responsible for the content and the writing of the paper. 


\section{Consent for publication}

Not applicable.

\section{Ethics approval and consent to participate}

The present research has been performed in accordance with the ethical standards of the 1964 Declaration of Helsinki and its later amendements. The Institutional Review Board of the Azienda Ospedaliero-Universitaria Ospeadali Riuniti Umberto I $^{\circ}$-Lancisi-Salesi granted retrospective access to the data without need for individual informed consent.

\section{Author details}

'Clinica Malattie Infettive; Università Politecnica delle Marche, Azienda Ospedaliero-Universitaria, Ospedali Riuniti Umberto Iº- G.M. Lancisi - G. Salesi, Ancona, Italy. ${ }^{2}$ Clinica Pediatrica; Università Politecnica delle Marche, Azienda Ospedaliero-Universitaria, Ospedali Riuniti Umberto Iº- G.M. Lancisi - G. Salesi, Ancona, Italy. ${ }^{3}$ Pediatria, Azienda Ospedaliero-Universitaria, Ospedali Riuniti Umberto I'- G.M. Lancisi - G. Salesi, Ancona, Italy. ${ }^{4}$ Anestesia e Rianimazione Pediatrica, Azienda Ospedaliero-Universitaria, Ospedali Riuniti Umberto $I^{\circ}$ G.M. Lancisi - G. Salesi, Ancona, Italy. ${ }^{5}$ Laboratorio di Microbiologia, Azienda Ospedaliero-Universitaria, Ospedali Riuniti Umberto I- G.M. Lancisi - G. Salesi, Ancona, Italy.

Received: 14 June 2016 Accepted: 11 July 2016

Published online: 09 August 2016

\section{References}

1. Cornely OA, Bassetti M, Calandra T, et al. ESCMID guideline for the diagnosis and management of Candida diseases 2012: non-neutropenic adult patients. Clin Microbiol Infect. 2012;18:19-37.

2. Lionakis MS, Netea MG. Candida and host determinants of susceptibility to invasive candidiasis. PLoS Pathog. 2013;9:e1003079.

3. Nieto MC, Tellería O, Cisterna R. Sentinel surveillance of invasive candidiasis in Spain: epidemiology and antifungal susceptibility. Diagn Microbiol Infect Dis. 2015;81:34-40.

4. Bassetti M, Righi E, Costa A, et al. Epidemiological trends in nosocomial candidemia in intensive care. BMC Infect Dis. 2006:6:21.

5. Barchiesi F, Orsetti E, Gesuita R, Skrami E, Manso E, Candidemia Study Group. Epidemiology, clinical characteristics, and outcome of candidemia in a tertiary referral center in Italy from 2010 to 2014 Infection. 2016:44:205-13.

6. Tortorano A, Prigitano A, Lazzarini C, et al. A 1-year prospective survey of candidemia in Italy and changing epidemiology over one decade. Infection. 2013;41:655-62.

7. Montagna MT, Caggiano G, Lovero G, et al. Epidemiology of invasive fungal infections in the intensive care unit: results of a multicenter Italian survey (AURORA Project). Infection. 2013;41:645-53.

8. Trofa D, Gàcser A, Nosanchuk JD. Candida parapsilosis, an emerging fungal pathogen. Clin Microbiol Rev. 2008;21:606-25.

9. Nucci M, Queiroz-Telles F, Alvarado-Matute T, et al. Epidemiology of candidemia in Latin America: a laboratory-based survey. PLoS One. 2013:8:e59373.

10. Cantòn $\mathrm{E}$, Pemàn J, Quindòs $\mathrm{G}$, et al. Prospective multicenter study of the epidemiology, molecular identification, and antifungal susceptibility of Candida parapsilosis, Candida orthopsilosis, and Candida metapsilosis isolated from patients with candidemia. Antimicrob Agents Chemother. 2011;55: 5590-6.

11. Borghi E, Sciota R, latta $R$, et al. Characterization of Candida parapsilosis complex strains isolated from invasive fungal infections. Eur J Clin Microbiol Infect Dis. 2011;30:1437-4.

12. Pfaller MA, Diekema DJ, Andes D, et al. Clinical breakpoints for the echinocandins and Candida revisited: integration of molecular, clinical, and microbiological data to arrive at species-specific interpretive criteria. Drug Resist Updat. 2011;14:164-76.

13. Pappas PG, CA K m, Andes D, et al. Clinical practice guidelines for the management of candidiasis: 2009 update by the Infectious Diseases Society of America. Clin Infect Dis. 2009;48:503-35

14. Chen CY, Sheng WH, Huang SY, et al. Clinical characteristics and treatment outcomes of patients with candidaemia due to Candida parapsilosis sensu lato species at a medical centre in Taiwan, 2000-12. J Antimicrob Chemother. 2015;70:1531-8.
15. Pfaller MA, Diekema DJ. Progress in antifungal susceptibility testing of Candida spp. by use of Clinical and Laboratory Standards Institute broth microdilution methods, 2010 to 2012. J Clin Microbiol. 2012;50:2846-56.

16. Andes DR, Safdar N, Baddley JW, et al. Impact of treatment strategy on outcomes in patients with candidemia and other forms of invasive candidiasis: a patient-level quantitative review of randomized trials. Clin Infect Dis. 2012;54:1110-22

17. Girmenia C, Martino P, De Bernardis F, et al. Rising incidence of Candida parapsilosis fungemia in patients with hematologic malignancies: clinical aspects, predisposing factors, and differential pathogenicity of the causative strains. Clin Infect Dis. 1996:23:506-14.

18. Miranda LD, Rodrigues EC, Costa SF, et al. Candida parapsilosis candidaemia in a neonatal unit over 7 years: a case series study. BMJ Open. 2012;2:4. 10. 1136/bmjopen-2012-000992

19. Almirante B, Rodríguez D, Cuenca-Estrella M, et al. Epidemiology, risk factors, and prognosis of Candida parapsilosis bloodstream infections: case-control population-based surveillance study of patients in Barcelona, Spain, from 2002 to 2003. J Clin Microbiol. 2006:44:1681-5.

20. Fernández-Ruiz M, Aguado JM, Almirante B, et al. Initial use of echinocandins does not negatively influence outcome in Candida parapsilosis bloodstream infection: a propensity score analysis. Clin Infect Dis. 2014:58:1413-21.

21. Fortún J, Martín-Dávila P, Gómez-García de la Pedrosa E, et al. Emerging trends in candidemia: a higher incidence but a similar outcome. J Infect. 2012;65:64-70

22. Wisplinghoff $\mathrm{H}$, Bischoff $\mathrm{T}$, Tallent SM, et al. Nosocomial bloodstream infections in US hospitals: analysis of 24,179 cases from a prospective nationwide surveillance study. Clin Infect Dis. 2004;39:309-17.

23. Bassetti M, Molinari MP, Mussap M, Viscoli C, Righi E. Candidemia in internal medicine departements: the burden of a rising problem. Clin Microbiol Infect. 2013:19:e281-4

24. Garey KW, Rege M, Pai MP, et al. Time to initiation of fluconazole therapy impacts mortality in patients with candidemia: a multi institutional study. Clin Infect Dis. 2006;43:25-31

25. Morrell M, Fraser VJ, Kollef MH. Delaying the empiric treatment of Candida bloodstream infection until positive blood culture results are obtained: a potential risk factor for hospital mortality. Antimicrob Agents Chemother. 2005:49:3640-5.

26. Kim SH, Yoon YK, Kim MJ, Sohn JW. Clinical impact of time to positivity for Candida species on mortality in patients with candidaemia. J Antimicrob Chemother. 2013;68:2890-7.

27. Mora-Duarte J, Betts R, Rotstein C, et al. Comparison of caspofungin and amphotericin B for invasive candidiasis. N Engl J Med. 2002;347:2020-9.

28. Reboli AC, Rotstein C, Pappas PG, et al. Anidulafungin versus fluconazole for invasive candidiasis. N Engl J Med. 2007;356:2472-82

29. Kuse ER, Chetchotisakd $P$, da Cunha CA, et al. Micafungin versus liposomal amphotericin B for candidaemia and invasive candidosis: a phase II randomised double-blind trial. Lancet. 2007:369:1519-27.

30. Pappas PG, Rotstein CM, Betts RF, et al. Micafungin versus caspofungin for treatment of candidemia and other forms of invasive candidiasis. Clin Infect Dis. 2007:45:883-93.

\section{Submit your next manuscript to BioMed Central and we will help you at every step:}

- We accept pre-submission inquiries

- Our selector tool helps you to find the most relevant journal

- We provide round the clock customer support

- Convenient online submission

- Thorough peer review

- Inclusion in PubMed and all major indexing services

- Maximum visibility for your research

Submit your manuscript at www.biomedcentral.com/submit 